\title{
MAPS WITH DIMENSIONALLY RESTRICTED FIBERS
}

\author{
VESKO VALOV
}

\begin{abstract}
We prove that if $f: X \rightarrow Y$ is a closed surjective map between metric spaces such that every fiber $f^{-1}(y)$ belongs to a class of space $\mathrm{S}$, then there exists an $F_{\sigma}$-set $A \subset X$ such that $A \in \mathrm{S}$ and $\operatorname{dim} f^{-1}(y) \backslash A=0$ for all $y \in Y$. Here, $\mathrm{S}$ can be one of the following classes: (i) $\{M$ : e $-\operatorname{dim} M \leq K\}$ for some $C W$-complex $K$; (ii) $C$-spaces; (iii) weakly infinite-dimensional spaces. We also establish that if $\mathrm{S}=\{M: \operatorname{dim} M \leq n\}$, then $\operatorname{dim} f \triangle g \leq 0$ for almost all $g \in C\left(X, \mathbb{I}^{n+1}\right)$.
\end{abstract}

\section{INTRODUCTION}

All spaces in the paper are assumed to be paracompact and all maps continuous. By $C(X, M)$ we denote all maps from $X$ into $M$. Unless stated otherwise, all function spaces are endowed with the source limitation topology provided $M$ is a metric space.

The paper is inspired by the results of Pasynkov [11], Torunczyk [15], Sternfeld [14] and Levin [8]. Pasynkov announced in [11] and proved in [12] that if $f: X \rightarrow Y$ is a surjective map with $\operatorname{dim} f \leq n$, where $X$ and $Y$ are finite-dimensional metric compacta, then $\operatorname{dim} f \triangle g \leq 0$ for almost all maps $g \in C\left(X, \mathbb{I}^{n}\right)$ (see [10] for a non-compact version of this result). Torunczyk [15] established (in a more general setting) that if $f, X$ and $Y$ are as in Pasynkov's theorem, then for each $0 \leq k \leq n-1$ there exists a $\sigma$-compact subset $A_{k} \subset X$ such that $\operatorname{dim} A_{k} \leq k$ and $\operatorname{dim} f \mid\left(X \backslash A_{k}\right) \leq n-k-1$.

Next results in this direction were established by Sternfeld and Levin. Sternfeld [14] proved that if in the cited above results $Y$ is not-necessarily finite-dimensional, then $\operatorname{dim} f \triangle g \leq 1$ for almost all $g \in C\left(X, \mathbb{I}^{n}\right)$ and there exists a $\sigma$-compact subset $A \subset X$ such that $\operatorname{dim} A \leq n-1$ and $\operatorname{dim} f \mid(X \backslash A) \leq 1$. Levin 8 improved Sternfeld's results by showing that $\operatorname{dim} f \triangle g \leq 0$ for almost all $g \in C\left(X, \mathbb{I}^{n+1}\right)$, and has shown that

2000 Mathematics Subject Classification. Primary 54F45; Secondary 54E40.

Key words and phrases. extensional dimension, $C$-spaces, 0-dimensional maps, metric compacta, weakly infinite-dimensional spaces.

The author was partially supported by NSERC Grant 261914-08. 
this is equivalent to the existence of an $n$-dimensional $\sigma$-compact subset $A \subset X$ with $\operatorname{dim} f \mid(X \backslash A) \leq 0$.

The above results of Pasynkov and Torunczyk were generalized in [17] for closed maps between metric space $X$ and $Y$ with $Y$ being a $C$-space (recall that each finite-dimensional paracompact is a $C$-space [6]). But the question whether the results of Pasynkov and Torunczyk remain valid without the finite-dimensionality assumption on $Y$ is still open.

In this paper we provide non-compact analogues of Levin's results for closed maps between metric spaces.

We say that a topological property of metrizable spaces is an Sproperty if the following conditions are satisfied: (i) $\mathrm{S}$ is hereditary with respect to closed subsets; (ii) if $X$ is metrizable and $\left\{H_{i}\right\}_{i=1}^{\infty}$ is a sequence of closed S-subsets of $X$, then $\bigcup_{i=1}^{\infty} H_{i} \in \mathrm{S}$; (iii) a metrizable space $X \in \mathrm{S}$ provided there exists a closed surjective map $f: X \rightarrow Y$ such that $Y$ is a 0 -dimensional metrizable space and $f^{-1}(y) \in \mathrm{S}$ for all $y \in Y$; (iv) any discrete union of S-spaces is an S-space.

Any map whose fibers have a given S-property is called an S-map.

Here are some examples of S-properties (we identify $\mathrm{S}$ with the class of spaces having the property $S$ ):

- $\mathrm{S}=\{X: \operatorname{dim} X \leq n\}$ for some $n \geq 0$;

- $\mathrm{S}=\left\{X: \operatorname{dim}_{G} X \leq n\right\}$, where $G$ is an Abelian group and $\operatorname{dim}_{G}$ is the cohomological dimension;

- more generally, $\mathrm{S}=\{X: \mathrm{e}-\operatorname{dim} X \leq K\}$, where $K$ is a $C W$ complex and e - dim is the extension dimension, see [4], 5];

- $\mathrm{S}=\{X: X$ is weakly infinite-dimensional $\}$;

- $\mathrm{S}=\{X: X$ is a $C$-space $\}$.

To show that the property e $-\operatorname{dim} \leq K$ satisfies condition (iii), we apply [3, Corollary 2.5]. For weakly infinite-dimensional spaces and $C$-spaces this follows from [7].

Theorem 1.1. Let $f: X \rightarrow Y$ be a closed surjective S-map with $X$ and $Y$ being metrizable spaces. Then there exists an $F_{\sigma}$-subset $A \subset X$ such that $A \in \mathrm{S}$ and $\operatorname{dim} f^{-1}(y) \backslash A=0$ for all $y \in Y$. Moreover, if $f$ is a perfect map, the conclusion remains true provided $\mathrm{S}$ is a property satisfying conditions $(i)-($ iii $)$.

Theorem 1.1 was established by Levin [9, Theorem 1.2] in the case $X$ and $Y$ are metric compacta and $\mathrm{S}$ is the property e $-\operatorname{dim} \leq K$ for a given $C W$-complex $K$. Levin's proof of this theorem remains valid for arbitrary S-property, but it doesn't work for non-compact spaces.

We say that a map $f: X \rightarrow Y$ has a countable functional weight (notation $W(f) \leq \aleph_{0}$ ), see [10]) if there exists a map $g: X \rightarrow \mathbb{I}^{\aleph_{0}}$ 
such that $f \triangle g: X \rightarrow Y \times \mathbb{1}^{\aleph_{0}}$ is an embedding. For example [12, Proposition 9.1], $W(f) \leq \aleph_{0}$ for any closed map $f: X \rightarrow Y$ such that $X$ is a metrizable space and every fiber $f^{-1}(y), y \in Y$, is separable.

Theorem 1.2. Let $X$ and $Y$ be paracompact spaces and $f: X \rightarrow Y$ a closed surjective map with $\operatorname{dim} f \leq n$ and $W(f) \leq \aleph_{0}$. Then $C\left(X, \mathbb{I}^{n+1}\right)$ equipped with the uniform convergence topology contains a dense subset of maps $g$ such that $\operatorname{dim} f \triangle g \leq 0$.

It was mentioned above that this corollary was established by Levin [8, Theorem 1.6] for metric compacta $X$ and $Y$. Levin's arguments don't work for non-compact spaces. We are using the Pasynkov's technique from [10] to reduced the proof of Theorem 1.2 to the case of $X$ and $Y$ being metric compacta.

Our last results concern the function spaces $C\left(X, \mathbb{I}^{n}\right)$ and $C\left(X, \mathbb{I}^{\aleph_{0}}\right)$ equipped with the source limitation topology. Recall that this topology on $C(X, M)$ with $M$ being a metrizable space can be described as follows: the neighborhood base at a given map $h \in C(X, M)$ consists of the sets $B_{\rho}(h, \epsilon)=\{g \in C(X, M): \rho(g, h)<\epsilon\}$, where $\rho$ is a fixed compatible metric on $M$ and $\epsilon: X \rightarrow(0,1]$ runs over continuous positive functions on $X$. The symbol $\rho(h, g)<\epsilon$ means that $\rho(h(x), g(x))<\epsilon(x)$ for all $x \in X$. It is well known that for paracompact spaces $X$ this topology doesn't depend on the metric $\rho$ and it has the Baire property provided $M$ is completely metrizable.

Theorem 1.3. Let $f: X \rightarrow Y$ be a perfect surjection between paracompact spaces and $W(f) \leq \aleph_{0}$.

(i) The maps $g \in C\left(X, \mathbb{I}^{\aleph_{0}}\right)$ such that $f \triangle g$ embeds $X$ into $Y \times$ $\mathbb{I}^{\aleph_{0}}$ form a dense $G_{\delta}$-set in $C\left(X, \mathbb{I}^{\aleph_{0}}\right)$ with respect to the source limitation topology;

(ii) If there exists a map $g \in C\left(X, \mathbb{I}^{n}\right)$ with $\operatorname{dim} f \triangle g \leq 0$, then all maps having this property form a dense $G_{\delta}$-set in $C\left(X, \mathbb{I}^{n}\right)$ with respect to the source limitation topology.

Corollary 1.4. Let $f: X \rightarrow Y$ be a perfect surjection with $\operatorname{dim} f \leq$ $n$ and $W(f) \leq \aleph_{0}$, where $X$ and $Y$ are paracompact spaces. Then all maps $g \in C\left(X, \mathbb{I}^{n+1}\right)$ with $\operatorname{dim} f \triangle g \leq 0$ form a dense $G_{\delta}$-set in $C\left(X, \mathbb{I}^{n+1}\right)$ with respect to the source limitation topology.

Corollary 1.4 follows directly from Theorem 1.2 and Theorem 1.3(ii). Corollary 1.5 below follows from Corollary 1.4 and [2, Corollary 1.1], see Section 3.

Corollary 1.5. Let $X, Y$ be paracompact spaces and $f: X \rightarrow Y$ a perfect surjection with $\operatorname{dim} f \leq n$ and $W(f) \leq \aleph_{0}$. Then for every matrizable ANR-space $M$ the maps $g \in C\left(X, \mathbb{I}^{n+1} \times M\right)$ such 
that $\operatorname{dim} g\left(f^{-1}(y)\right) \leq n+1$ for all $y \in Y$ form a dense $G_{\delta}$-set $E$ in $C\left(X, \mathbb{I}^{n+1} \times M\right)$ with respect to the source limitation topology.

Finally, let us formulate the following question concerning property $\mathrm{S}$ (an affirmative answer of this question yields that (strong) countabledimensionality is an S-property):

Question 1.6. Suppose $f: X \rightarrow Y$ is a perfect surjection between metrizable spaces such that $\operatorname{dim} Y=0$ and each fiber $f^{-1}(y), y \in$ $Y$, is (strongly) countable-dimensional. Is it true that $X$ is (strongly) countable-dimensional?

\section{S-PROPERTIES AND MAPS INTO FINITE-DIMENSIONAL CUBES}

This section contains the proofs of Theorem 1.1 and Theorem 1.2.

Proof of Theorem 1.1. We follow the proof of [18, Proposition 4.1]. Let us show first that the proof is reduced to the case $f$ is a perfect map. Indeed, according to Vainstein's lemma, the boundary $\operatorname{Fr} f^{-1}(y)$ of every fiber $f^{-1}(y)$ is compact. Defining $F(y)$ to be $\operatorname{Fr} f^{-1}(y)$ if $\operatorname{Fr} f^{-1}(y) \neq \varnothing$, and an arbitrary point from $f^{-1}(y)$ otherwise, we obtain a set $X_{0}=\bigcup\{F(y): y \in Y\}$ such that $X_{0} \subset X$ is closed and the restriction $f \mid X_{0}$ is a perfect map. Moreover, each $f^{-1}(y) \backslash X_{0}$ is open in $X$ and has the property $\mathrm{S}$ (as an $F_{\sigma}$-subset of the S-space $f^{-1}(y)$ ). Hence, $X \backslash X_{0}$ being the union of the discrete family $\left\{f^{-1}(y) \backslash X_{0}: y \in Y\right\}$ of S-set is an S-set. At the same time $X \backslash X_{0}$ is open in $X$. Consequently, $X \backslash X_{0}$ is the union of countably many closed sets $X_{i} \subset X, i=1,2, \ldots$ Obviously, each $X_{i}, i \geq 1$, also has the property S. Therefore, it suffices to prove Theorem 1.1 for the S-map $f \mid X_{0}: X_{0} \rightarrow Y$.

So, we may suppose that $f$ is perfect. According to [10], there exists a map $g: X \rightarrow \mathbb{I}^{\aleph_{0}}$ such that $g$ embeds every fiber $f^{-1}(y), y \in Y$. Let $g=\triangle_{i=1}^{\infty} g_{i}$ and $h_{i}=f \triangle g_{i}: X \rightarrow Y \times \mathbb{I}, i \geq 1$. Moreover, we choose countably many closed intervals $\mathbb{I}_{j}$ such that every open subset of $\mathbb{I}$ contains some $\mathbb{I}_{j}$. By [17, Lemma 4.1], for every $j$ there exists a 0-dimensional $F_{\sigma}$-set $C_{j} \subset Y \times \mathbb{I}_{j}$ such that $C_{j} \cap\left(\{y\} \times \mathbb{I}_{j}\right) \neq \varnothing$ for every $y \in Y$. Now, consider the sets $A_{i j}=h_{i}^{-1}\left(C_{j}\right)$ for all $i, j \geq 1$ and let $A$ be their union. Since $f$ is an S-map, so is the map $h_{i}$ for any $i$. Hence, $A_{i j}$ has property $\mathrm{S}$ for all $i, j$. This implies that $A$ has also the same property.

It remains to show that $\operatorname{dim} f^{-1}(y) \backslash A \leq 0$ for every $y \in Y$. Let $\operatorname{dim} f^{-1}\left(y_{0}\right) \backslash A>0$ for some $y_{0}$. Since $g \mid f^{-1}\left(y_{0}\right)$ is an embedding, there exists an integer $i$ such that $\operatorname{dim} g_{i}\left(f^{-1}\left(y_{0}\right) \backslash A\right)>0$. Then $g_{i}\left(f^{-1}\left(y_{0}\right) \backslash A\right)$ has a nonempty interior in $\mathbb{I}$. So, $g_{i}\left(f^{-1}\left(y_{0}\right) \backslash A\right)$ contains some $\mathbb{I}_{j}$. Choose $t_{0} \in \mathbb{I}_{j}$ with $c_{0}=\left(y_{0}, t_{0}\right) \in C_{j}$. Then there exists $x_{0} \in$ 
$f^{-1}\left(y_{0}\right) \backslash A$ such that $g_{i}\left(x_{0}\right)=t_{0}$. On the other hand, $x_{0} \in h_{i}^{-1}\left(c_{0}\right) \subset$ $A_{i j} \subset A$, a contradiction.

Proof of Theorem 1.2. We first prove next proposition which is a small modification of [10, Theorem 8.1]. For any map $f: X \rightarrow Y$ we consider the set $C\left(X, Y \times \mathbb{I}^{n+1}, f\right)$ consisting of all maps $g: X \rightarrow$ $Y \times \mathbb{I}^{n+1}$ such that $f=\pi_{n} \circ g$, where $\pi_{n}: Y \times \mathbb{I}^{n+1} \rightarrow Y$ is the projection onto $Y$. We also consider the other projection $\varpi_{n}: Y \times \mathbb{I}^{n+1} \rightarrow \mathbb{I}^{n+1}$. It is easily seen that the formula $g \rightarrow \varpi_{n} \circ g$ provides one-to-one correspondence between $C\left(X, Y \times \mathbb{I}^{n+1}, f\right)$ and $C\left(X, \mathbb{I}^{n+1}\right)$. So, we may assume that $C\left(X, Y \times \mathbb{I}^{n+1}, f\right)$ is a metric space isometric with $C\left(X, \mathbb{I}^{n+1}\right)$, where $C\left(X, \mathbb{I}^{n+1}\right)$ is equipped with the supremum metric.

Proposition 2.1. Let $f: X \rightarrow Y$ be an $n$-dimensional surjective map between compact spaces with $n>0$ and $\lambda: X \rightarrow Z$ a map into a metric compactum $Z$. Then the maps $g \in C\left(X, Y \times \mathbb{I}^{n+1}, f\right)$ satisfying the condition below form a dense subset of $C\left(X, Y \times \mathbb{I}^{n+1}, f\right)$ : there exists a compact space $H$ and maps $\varphi: X \rightarrow H, h: H \rightarrow Y \times \mathbb{I}^{n+1}$ and $\mu: H \rightarrow Z$ such that $\lambda=\mu \circ \varphi, g=h \circ \varphi, W(h) \leq \aleph_{0}$ and $\operatorname{dim} h=0$.

Proof. We fix a map $g_{0} \in C\left(X, Y \times \mathbb{I}^{n+1}, f\right)$ and $\epsilon>0$. Let $g_{1}=$ $\varpi_{n} \circ g_{0}$. Then $\lambda \triangle g_{1} \in C\left(X, Z \times \mathbb{I}^{n+1}\right)$. Consider also the constant maps $f^{\prime}: Z \times \mathbb{I}^{n+1} \rightarrow P t$ and $\eta: Y \rightarrow P t$, where $P t$ is the one-point space. So, we have $\eta \circ f=f^{\prime} \circ\left(\lambda \triangle g_{1}\right)$. According to Pasynkov's factorization theorem [13, Theorem 13], there exist metrizable compacta $K, T$ and maps $f^{*}: K \rightarrow T, \xi_{1}: X \rightarrow K, \xi_{2}: K \rightarrow Z \times \mathbb{I}^{n+1}$ and $\eta^{*}: Y \rightarrow T$ such that:

- $\eta^{*} \circ f=f^{*} \circ \xi_{1}$

- $\xi_{2} \circ \xi_{1}=\lambda \triangle g_{1}$

- $\operatorname{dim} f^{*} \leq \operatorname{dim} f \leq n$.

If $p: Z \times \mathbb{I}^{n+1} \rightarrow Z$ and $q: Z \times \mathbb{I}^{n+1} \rightarrow \mathbb{I}^{n+1}$ denote the corresponding projections, we have

$$
p \circ \xi_{2} \circ \xi_{1}=\lambda \text { and } q \circ \xi_{2} \circ \xi_{1}=g_{1} .
$$

Since $\operatorname{dim} f^{*} \leq n$, by Levin's result [8, Theorem 1.6], there exists a $\operatorname{map} \phi: K \rightarrow \mathbb{I}^{n+1}$ such that $\phi$ is $\epsilon$-close to $q \circ \xi_{2}$ and $\operatorname{dim} f^{*} \triangle \phi \leq 0$. Then the map $\phi \circ \xi_{1}$ is $\epsilon$-close to $g_{1}$, so $g=f \triangle\left(\phi \circ \xi_{1}\right)$ is $\epsilon$-close to $g_{0}$. Denote $\varphi=f \triangle \xi_{1}, H=\varphi(X)$ and $h=\left(i d_{Y} \times \phi\right) \mid H$. If $\varpi_{H}: H \rightarrow K$ is the restriction of the projection $Y \times K \rightarrow K$ on $H$, we have

$\lambda=p \circ \xi_{2} \circ \xi_{1}=p \circ \xi_{2} \circ \varpi_{H} \circ \varphi$, so $\lambda=\mu \circ \varphi$, where $\mu=p \circ \xi_{2} \circ \varpi_{H}$. Moreover, $g=f \triangle\left(\phi \circ \xi_{1}\right)=\left(i d_{Y} \times \phi\right) \circ\left(f \triangle \xi_{1}\right)=h \circ \varphi$. Since $K$ is a metrizable compactum, $W(\phi) \leq \aleph_{0}$. Hence, $W(h) \leq \aleph_{0}$. 
To show that $\operatorname{dim} h \leq 0$, it suffices to prove that $\operatorname{dim} h \leq \operatorname{dim} f^{*} \triangle \phi$. To this end, we show that any fiber $h^{-1}((y, v))$, where $(y, v) \in Y \times \mathbb{I}^{n+1}$, is homeomorphic to a subset of the fiber $\left(f^{*} \triangle \phi\right)^{-1}\left(\left(\eta^{*}(y), v\right)\right)$. Indeed, let $\pi_{Y}$ be the restriction of the projection $Y \times K \rightarrow Y$ on the set $H$. Since $\eta^{*} \circ f=f^{*} \circ \xi_{1}, H$ is a subset of the pullback of $Y$ and $K$ with respect to the maps $\eta^{*}$ and $f^{*}$. Therefore, $\varpi_{H}$ embeds every fiber $\pi_{Y}^{-1}(y)$ into $\left(f^{*}\right)^{-1}(y), y \in Y$. Let $a_{i}=\left(y_{i}, k_{i}\right) \in H \subset Y \times K$, $i=1,2$, such that $h\left(a_{1}\right)=h\left(a_{2}\right)$. Then $\left(y_{1}, \phi\left(k_{1}\right)\right)=\left(y_{2}, \phi\left(k_{2}\right)\right)$, so $y_{1}=y_{2}=y$ and $\phi\left(k_{1}\right)=\phi\left(k_{2}\right)=v$. This implies $\varpi_{H}\left(a_{i}\right)=$ $k_{i} \in\left(f^{*}\right)^{-1}\left(\eta^{*}\left(\pi_{Y}\left(a_{i}\right)\right)\right)=\left(f^{*}\right)^{-1}\left(\eta^{*}(y)\right), i=1,2$. Hence, $\varpi_{H}$ embeds the fiber $h^{-1}((y, v))$ into the fiber $\left(f^{*} \triangle \phi\right)^{-1}\left(\left(\eta^{*}(y), v\right)\right)$. Consequently, $\operatorname{dim} h \leq \operatorname{dim} f^{*} \triangle \phi=0$.

We can prove now Theorem 1.2. It suffices to show every map from $C\left(X, Y \times \mathbb{I}^{n+1}, f\right)$ can be approximated by maps $g \in C\left(X, Y \times \mathbb{I}^{n+1}, f\right)$ with $\operatorname{dim} g \leq 0$. We fix $g_{0} \in C\left(X, Y \times \mathbb{I}^{n+1}, f\right)$ and $\epsilon>0$. Since $W(f) \leq \aleph_{0}$, there exists a map $\lambda: X \rightarrow \mathbb{I}^{\aleph_{0}}$ such that $f \triangle \lambda$ is an embedding. Let $\beta f: \beta X \rightarrow \beta Y$ be the Cech-Stone extension of the map $f$. Then $\operatorname{dim} \beta f \leq n$, see [13, Theorem 15]. Consider also the maps $\beta \lambda: \beta X \rightarrow \mathbb{I}^{\aleph_{0}}$ and $\bar{g}_{0}=\beta f \triangle \beta g_{1}$, where $g_{1}=\varpi_{n} \circ g_{0}$. According to Proposition 2.1, there exists a map $\bar{g} \in C\left(\beta X, \beta Y \times \mathbb{I}^{n+1}, \beta f\right)$ which is $\epsilon$-close to $\bar{g}_{0}$ and satisfies the following conditions: there exists a compact space $H$ and maps $\varphi: \beta X \rightarrow H, h: H \rightarrow \beta Y \times \mathbb{I}^{n+1}$ and $\mu: H \rightarrow \mathbb{I}^{\aleph_{0}}$ such that $\beta \lambda=\mu \circ \varphi, \bar{g}=h \circ \varphi, W(h) \leq \aleph_{0}$ and $\operatorname{dim} h=0$. We have the following equalities

$\beta f \triangle \beta \lambda=\left(\pi_{n} \circ \bar{g}\right) \triangle(\mu \circ \varphi)=\left(\pi_{n} \circ h \circ \varphi\right) \triangle(\mu \circ \varphi)=\left(\left(\pi_{n} \circ h\right) \triangle \mu\right) \circ \varphi$, where $\pi_{n}$ denotes the projection $\beta Y \times \mathbb{I}^{n+1} \rightarrow \beta Y$. This implies that $\varphi$ embeds $X$ into $H$ because $f \triangle \lambda$ embeds $X$ into $Y \times \mathbb{I}^{\aleph_{0}}$. Let $g$ be the restriction of $\bar{g}$ over $X$. Identifying $X$ with $\varphi(X)$, we obtain that $h$ is an extension of $g$. Hence, $\operatorname{dim} g \leq \operatorname{dim} h=0$. Observe also that $g$ is $\epsilon$-close to $g_{0}$, which completes the proof.

\section{Proof of Theorem 1.3 And Corollary 1.5}

Proof of Theorem 1.3(ii). We first prove condition (ii). Since $W(f) \leq$ $\aleph_{0}$, there exists a map $\lambda: X \rightarrow \mathbb{I}^{\aleph_{0}}$ such that $f \triangle \lambda$ embeds $X$ into $Y \times$ $\mathbb{I}^{\aleph_{0}}$. Choose a sequence $\left\{\gamma_{k}\right\}_{k \geq 1}$ of open covers of $\mathbb{I}^{\aleph_{0}}$ with $\operatorname{mesh}\left(\gamma_{k}\right) \leq$ $1 / k$, and let $\omega_{k}=\lambda^{-1}\left(\gamma_{k}\right)$ for all $k$. We denote by $C_{\left(\omega_{k}, 0\right)}\left(X, \mathbb{I}^{n}, f\right)$ the set of all maps $g \in C\left(X, \mathbb{I}^{n}\right)$ with the following property: every $z \in(f \triangle g)(X)$ has a neighborhood $V_{z}$ in $Y \times \mathbb{I}^{n}$ such that $(f \triangle g)^{-1}\left(V_{z}\right)$ can be represented as the union of a disjoint open in $X$ family refining the cover $\omega_{k}$. According to [17, Lemma 2.5], each of the sets 
$C_{\left(\omega_{k}, 0\right)}\left(X, \mathbb{I}^{n}, f\right), k \geq 1$, is open in $C\left(X, \mathbb{I}^{n}\right)$ with respect to the source limitation topology. It follows from the definition of the covers $\omega_{k}$ that $\bigcap_{k>1} C_{\left(\omega_{k}, 0\right)}\left(X, \mathbb{I}^{n}, f\right)$ consists of maps $g$ with $\operatorname{dim} f \triangle g \leq 0$. Since $C\left(X, \mathbb{I}^{n}\right)$ with the source limitation topology has the Baire property, it remains to show that any $C_{\left(\omega_{k}, 0\right)}\left(X, \mathbb{I}^{n}, f\right)$ is dense in $C\left(X, \mathbb{I}^{n}\right)$.

To this end, we fix a cover $\omega_{m}$, a map $g_{0} \in C\left(X, \mathbb{I}^{n}\right)$ and a function $\epsilon: X \rightarrow(0,1]$. We are going to find $h \in C_{\left(\omega_{m}, 0\right)}\left(X, \mathbb{I}^{n}, f\right)$ such that $\rho\left(g_{0}(x), h(x)\right)<\epsilon(x)$ for all $x \in X$, where $\rho$ is the Euclidean metric on $\mathbb{I}^{n}$. Then, by [1, Lemma 8.1], there exists an open cover $\mathcal{U}$ of $X$ satisfying the following condition: if $\alpha: X \rightarrow K$ is a $\mathcal{U}$-map into a paracompact space $K$ (i.e., $\alpha^{-1}(\omega)$ refines $\mathcal{U}$ for some open cover $\omega$ of $K)$, then there exists a map $q: G \rightarrow \mathbb{I}^{n}$, where $G$ is an open neighborhood of $\overline{\alpha(X)}$ in $K$, such that $g_{0}$ and $q \circ \alpha$ are $\epsilon / 2$-close with respect to the metric $\rho$. Let $\mathcal{U}_{1}$ be an open cover of $X$ refining both $\mathcal{U}$ and $\omega_{m}$ such that $\inf \{\epsilon(x): x \in U\}>0$ for all $U \in \mathcal{U}_{1}$.

Since $\operatorname{dim} f \triangle g \leq 0$ for some $g \in C\left(X, \mathbb{I}^{n}\right)$, according to [1, Theorem $6]$ there exists an open cover $\mathcal{V}$ of $Y$ such that for any $\mathcal{V}$-map $\beta: Y \rightarrow L$ into a simplicial complex $L$ we can find a $\mathcal{U}_{1}$-map $\alpha: X \rightarrow K$ into a simplicial complex $K$ and a perfect $P L$-map $p: K \rightarrow L$ with $\beta \circ f=p \circ \alpha$ and $\operatorname{dim} p \leq n$. We can assume that $\mathcal{V}$ is locally finite. Take $L$ to be the nerve of the cover $\mathcal{V}$ and $\beta: Y \rightarrow L$ the corresponding natural map. Then there exist a simplicial complex $K$ and maps $p$ and $\alpha$ satisfying the above conditions. Hence, the following diagram is commutative.

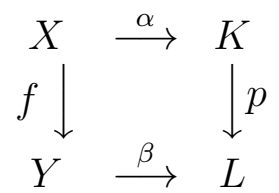

Since $K$ is paracompact, the choice of the cover $\mathcal{U}$ guarantees the existence of a map $\varphi: G \rightarrow \mathbb{I}^{n}$, where $G \subset K$ is an open neighborhood of $\overline{\alpha(X)}$, such that $g_{0}$ and $h_{0}=\varphi \circ \alpha$ are $\epsilon / 2$-close with respect to $\rho$. Replacing the triangulation of $K$ by a suitable subdivision, we may additionally assume that no simplex of $K$ meets both $\overline{\alpha(X)}$ and $K \backslash G$. So, the union $N$ of all simplexes $\sigma \in K$ with $\sigma \cap \overline{\alpha(X)} \neq \varnothing$ is a subcomplex of $K$ and $N \subset G$. Moreover, since $N$ is closed in $K$, $p_{N}=p \mid N: N \rightarrow L$ is a perfect map. Therefore, we have the following commutative diagram: 


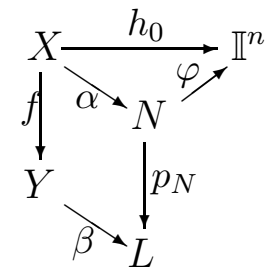

Using that $\alpha$ is a $\mathcal{U}_{1}$-map and $\inf \{\epsilon(x): x \in U\}>0$ for all $U \in$ $\mathcal{U}_{1}$, we can construct a continuous function $\epsilon_{1}: N \rightarrow(0,1]$ and an open cover $\gamma$ of $N$ such that $\epsilon_{1} \circ \alpha \leq \epsilon$ and $\alpha^{-1}(\gamma)$ refines $\mathcal{U}_{1}$. Since $\operatorname{dim} p_{N} \leq \operatorname{dim} p \leq n$ and $L$, being a simplicial complex, is a $C$-space, we can apply [17, Theorem 2.2] to find a map $\varphi_{1} \in C_{(\gamma, 0)}\left(N, \mathbb{I}^{n}, p_{N}\right)$ which is $\epsilon_{1} / 2$-close to $\varphi$. Let $h=\varphi_{1} \circ \alpha$. Then $h$ and $h_{0}$ are $\epsilon / 2$-close because $\epsilon_{1} \circ \alpha \leq \epsilon$. On the other hand, $h_{0}$ is $\epsilon / 2$-close to $g_{0}$. Hence, $g_{0}$ and $h$ are $\epsilon$-close.

It remains to show that $h \in C_{\left(\omega_{m}, 0\right)}\left(X, \mathbb{I}^{n}, f\right)$. To this end, fix a point $z=(f(x), h(x)) \in(f \triangle h)(X) \subset Y \times \mathbb{I}^{n}$ and let $y=f(x)$. Then $w=\left(p_{N} \triangle \varphi_{1}\right)(\alpha(x))=(\beta(y), h(x))$. Since $\varphi_{1} \in C_{(\gamma, 0)}\left(N, \mathbb{I}^{n}, p_{N}\right)$, there exists a neighborhood $V_{w}$ of $w$ in $L \times \mathbb{I}^{n}$ such that $W=\left(p_{N} \triangle \varphi_{1}\right)^{-1}\left(V_{w}\right)$ is a union of a disjoint open family in $N$ refining $\gamma$. We can assume that $V_{w}=V_{\beta(y)} \times V_{h(x)}$, where $V_{\beta(y)}$ and $V_{h(x)}$ are neighborhoods of $\beta(y)$ and $h(x)$ in $Y$ and $\mathbb{I}^{n}$, respectively. Consequently, $(f \triangle h)^{-1}(\Gamma)=$ $\alpha^{-1}(W)$, where $\Gamma=\beta^{-1}\left(V_{\beta(y)}\right) \times V_{h(x)}$. Finally, observe that $\alpha^{-1}(W)$ is a disjoint union of an open in $X$ family refining $\omega_{m}$. Therefore, $h \in C_{\left(\omega_{m}, 0\right)}\left(X, \mathbb{I}^{n}, f\right)$.

Proof of Theorem 1.3( $i)$. Let $\lambda$ and $\omega_{k}$ be as in the proof of Theorem 1.3(i). Denote by $C_{\omega_{k}}\left(X, \mathbb{I}^{\aleph_{0}}, f\right)$ the set of all $g \in C\left(X, \mathbb{I}^{\aleph_{0}}\right)$ such that $f \triangle g$ is an $\omega_{k}$-map. It can be shown that every $C_{\omega_{k}}\left(X, \mathbb{I}^{\aleph_{0}}, f\right)$ is open in $C\left(X, \mathbb{I}^{\aleph_{0}}\right)$ with the source limitation topology (see [16, Proposition 3.1]). Moreover, $\bigcap_{k \geq 1} C_{\omega_{k}}\left(X, \mathbb{I}^{\aleph_{0}}, f\right)$ consists of maps $g$ with $f \triangle g$ embedding $X$ into $Y \times \mathbb{I}^{\aleph_{0}}$. So, we need to show that each $C_{\omega_{k}}\left(X, \mathbb{I}^{\aleph_{0}}, f\right)$ is dense in $C\left(X, \mathbb{I}^{\aleph_{0}}\right)$ equipped with the source limitation topology.

To prove this fact we follow the notations and the arguments from the proof of Theorem 1.3(ii) (that $C_{\left(\omega_{k}, 0\right)}\left(X, \mathbb{I}^{n}, f\right)$ are dense in $C\left(X, \mathbb{I}^{n}\right)$ ) by considering $\mathbb{I}^{\aleph_{0}}$ instead of $\mathbb{I}^{n}$. We fix a cover $\omega_{m}$, a map $g_{0} \in C\left(X, \mathbb{I}^{\aleph_{0}}\right)$ and a function $\epsilon \in C(X,(0,1])$. Since $W(f) \leq \aleph_{0}$, we can apply Theorem 6 from [1] to find an open cover $\mathcal{V}$ of $Y$ such that for any $\mathcal{V}$-map $\beta: Y \rightarrow L$ into a simplicial complex $L$ there exists a $\mathcal{U}_{1}$-map $\alpha: X \rightarrow K$ into a simplicial complex $K$ and a perfect $P L$-map $p: K \rightarrow$ $L$ with $\beta \circ f=p \circ \alpha$. Proceeding as before, we find a map $h=\varphi_{1} \circ \alpha$ which is $\epsilon$-close to $g_{0}$, where $\varphi_{1} \in C_{\gamma}\left(N, \mathbb{I}^{\aleph_{0}}, p_{N}\right)$. It is easily seen that 
$\varphi_{1} \in C_{\gamma}\left(N, \mathbb{I}^{\aleph_{0}}, p_{N}\right)$ implies $h \in C_{\omega_{m}}\left(X, \mathbb{I}^{\aleph_{0}}, f\right)$. So, $C_{\omega_{m}}\left(X, \mathbb{I}^{\aleph_{0}}, f\right)$ is dense in $C\left(X, \mathbb{I}^{\aleph_{0}}\right)$.

Proof of Corollary 1.5. It follows from [2, Proposition 2.1] that the set $E$ is $G_{\delta}$ in $C\left(X, \mathbb{I}^{n+1} \times M\right)$. So, we need to show it is dense in $C\left(X, \mathbb{I}^{n+1} \times M\right)$. To this end, we fix $g^{0}=\left(g_{1}^{0}, g_{2}^{0}\right) \in C\left(X, \mathbb{I}^{n+1} \times M\right)$ with $g_{1}^{0} \in C\left(X, \mathbb{I}^{n+1}\right)$ and $g_{2}^{0} \in C(X, M)$. Since, by Corollary 1.4 , the set

$$
G_{1}=\left\{g_{1} \in C\left(X, \mathbb{I}^{n+1}\right): \operatorname{dim} f \triangle g_{1} \leq 0\right\}
$$

is dense in $C\left(X, \mathbb{I}^{n+1}\right)$, we may approximate $g_{1}^{0}$ by a map $h_{1} \in G_{1}$. Then, according to [2, Corollary 1.1], the maps $g_{2} \in C(X, M)$ with $\operatorname{dim} g_{2}\left(\left(f \triangle h_{1}\right)^{-1}(z)\right)=0$ for all $z \in Y \times \mathbb{I}^{n+1}$ form a dense subset $G_{2}$ of $C(X, M)$. So, we can approximate $g_{2}^{0}$ by a map $h_{2} \in G_{2}$. Let us show that the map $h=\left(h_{1}, h_{2}\right) \in C\left(X, \mathbb{I}^{n+1}\right) \times M$ belongs to $E$. We define the map $\pi_{h}:(f \triangle h)(X) \rightarrow\left(f \triangle h_{1}\right)(X), \pi_{h}\left(f(x), h_{1}(x), h_{2}(x)\right)=$ $\left(f(x), h_{1}(x)\right), x \in X$. Because $f$ is perfect, so is $\pi_{h}$. Moreover, $\left(\pi_{h}\right)^{-1}\left(f(x), h_{1}(x)\right)=h_{2}\left(f^{-1}(f(x)) \cap h_{1}^{-1}\left(h_{1}(x)\right)\right), x \in X$. So, every fiber of $\pi_{h}$ is 0-dimensional. We also observe that $\pi_{h}\left(h\left(f^{-1}(y)\right)\right)=$ $\left(f \triangle h_{1}\right)\left(f^{-1}(y)\right)$ and the restriction $\pi_{h} \mid h\left(f^{-1}(y)\right)$ is a perfect surjection between the compact spaces $h\left(f^{-1}(y)\right)$ and $\left(f \triangle h_{1}\right)\left(f^{-1}(y)\right)$ for any $y \in$ $Y$. Since $\left(f \triangle h_{1}\right)\left(f^{-1}(y)\right) \subset\{y\} \times \mathbb{I}^{n+1}, \operatorname{dim}\left(f \triangle h_{1}\right)\left(f^{-1}(y)\right) \leq n+1$,

$y \in Y$. Consequently, applying the Hurewicz's dimension-lowering theorem [6] for the map $\pi_{h} \mid h\left(f^{-1}(y)\right)$, we have $\operatorname{dim} h\left(f^{-1}(y)\right) \leq n+1$. Therefore, $h \in E$, which completes the proof.

\section{REFERENCES}

1. T. Banakh and V. Valov, General Position Properties in Fiberwise Geometric Topology, arXiv:math.GT/10012522.

2. T. Banakh and V. Valov, Spaces with fibered approximation property in dimension n, Central European J. Math. 8:3 (2010), 411-420.

3. A. Chigogidze and V. Valov, Extension dimension and $C$-spaces, Bull. London Math. Soc., 34, 6 (2002), 708-716.

4. A. N. Dranishnikov, The Eilenberg-Borsuk theorem for mappings into an arbitrary complex, Russian Acad. Sci. Sb. Math. 81:2 (1995), 467-475.

5. A. Dranishnikov and J. Dydak, Extension dimension and extension types, Proc. Steklov Inst. Math. 212, (1996), 55-88.

6. R. Engelking, Theory of Dimensions: Finite and Infinitite, Heldermann, Lemgo, 1995.

7. Y. Hattori and K. Yamada, Closed pre-images of $C$-spaces, Math. Japonica 34:4 (1989), 555-561.

8. M. Levin, Bing maps and finite-dimensional maps, Fund. Math. 151:1 (1996), $47-52$.

9. M. Levin, On extensional dimension of maps, Topol. Appl. 103 (2000), 33-35. 
10. B. Pasynkov, On geometry of continuous maps of countable functional weight, Fundam. Prikl. Matematika 4:1 (1998), 155-164 (in Russian).

11. B. Pasynkov, On dimension and geometry of mappings, Dokl. Akad. Nauk SSSR 221 (1975), 543-546 (in Russian).

12. B. Pasynkov, On geometry of continuous maps of finite-dimensional compact metric spaces, Trudy Mat. Inst. Steklov 212 (1996), 155-164 (in Russian).

13. B. Pasynkov, Factorization theorems in dimension theory, Uspekhi Mat. Nauk 36:3 (1981), 147-175 (in Russian).

14. Y. Sternfeld, On finite-dimensional maps and other maps with "small" fibers, Fund. Math. 147 (1995), 127-133.

15. H. Torunczyk, Finite-to-one restrictions of continuous functions, Fund. Math. 75 (1985), 237-249.

16. M. Tuncali and V. Valov, On finite-dimensional maps, Tsukuba J. Math. 28:1, (2004), 155-167.

17. M. Tuncali and V. Valov, On dimensionally restricted maps, Fund. Math. 175:1, (2002), no. 1, 35-52.

18. V. Valov, Parametric Bing and Krasinkiewicz maps:revisited, Proc. Amer. Math. Soc. 139:2 (2011), 747-756.

Department of Computer Science and Mathematics, Nipissing University, 100 College Drive, P.O. Box 5002, North Bay, ON, P1B 8L7, CANADA

E-mail address: veskov@nipissingu.ca 